\title{
From the Popularization of Mandarin to Talk About the Inheritance of Dialect -To Take Maanshan as an Example
}

\author{
Yue Chen ${ }^{1}$ \\ ${ }^{1}$ Taizhou College, Nanjing Normal University, Taizhou, Jiangsu, China \\ Correspondence: Yue Chen, Associate Professor, Associate Dean, School of Humanities and Media, Taizhou \\ College, Nanjing Normal University, Taizhou College, Nanjing Normal University, Taizhou 225300, Jiangsu, \\ China.E-mail: 110186712@qq.com
}

Received: December 19, 2021

Accepted: January 15, 2022

Online Published: January 26, 2022

doi:10.20849/ajsss.v7i1.991

URL: https://doi.org/10.20849/ajsss.v7i1.991

\begin{abstract}
Maanshan, abbreviated as "Ma city", is located in the east of Anhui. It is the intersection of Jiangsu and Anhui, and is also the core of Hefei metropolitan area and Nanjing metropolitan area. With the rapid development of urbanization in China and the popularization of Mandarin, the collision between dialect and Mandarin is more extensive. They interact, influence and connect with each other, forming a unique scenic. This article is based on the contact between Maanshan dialect and Mandarin, to understand the phenomenon and characteristics of the collision between Maanshan dialect and Mandarin in the process of urbanization, and how to make Mandarin coexist harmoniously with dialects, and how to promote Mandarin better while respecting dialects.
\end{abstract}

Keywords: Mandarin, universal, dialect, inherit, Maanshan

\section{Introduction}

As we all know, Mandarin is the standard language of modern Chinese, with Beijing voice as the standard voice, Northern Dialect as the basic dialect, and the typical mondern vernacular works as the grammar standard. Dialect, is a language different from the standard language, has a strong region, mainly the language spoken in people's daily life. There are certain regional language changes between Mandarin and dialects. Dialects vary greatly from region to region, which brings a lot of inconvenience to people's communication. Therefore, it is imperative to vigorously promote the nationwide universal Mandarin. But dialect and Mandarin are also not opposite relations, but should be an equal language communication. Dialect is often a symbol of a local cultural history and culture, representing the different cultures of each region. Therefore, in the background of vigorously promoting Mandarin, it's also essential for us to protect, develop and inherit dialects.

\section{The Popularization of Mandarin}

\subsection{Popularization and Significance of Mandarin}

In September 2017, the State Language Commission and the Ministry of Education issued a series of documents, such as the Development Report of the Chinese Language and Writing Industry, which comprehensively and systematically demonstrated its development status and work achievements in the language and writing industry. Data show that the penetration rate of Mandarin Chinese increased from 53\% in 2000 to about 73\% in 2015, and the proportion of literate people using standard Chinese characters was more than $95 \%$. Mandarin is essential in our daily life. If you go to other places, everyone speaks to you in dialect, you can not understand, then this time Mandarin can come in handy, with it can well solve the problem in work and study. China is a country with multi-ethnic groups and multiple dialects. By increasing the penetration rate of Mandarin, all ethnic groups can have a "common language" that they can understand. As today's society is constantly developing and advancing, when people from various provinces and regions communicate and learn from each other, they can have a common language, which is conducive to enhancing the communication between all ethnic groups and regions, to maintaining national unity and unity, to promoting social civilization and progress, and enhancing the cohesion of the Chinese nation.

\subsection{The Popularization of Mandarin in Maanshan}

Maanshan is a city of mixed ethnic minorities. The survey shows that by the end of 2012, there were 43 ethnic minorities and a population of nearly 20,000. In Zheng Port District, Bowang District and He County, there are 
three ethnic villages and one ethnic primary school. Maanshan city has a Hui population of about 13,000, accounting for $65 \%$ of the total ethnic minority population. Of them, $66.31 \%$ can communicate with people in the Maanshan dialect, and 53.3\% in Mandarin. This figure showed a 5\% increase in the number of people using Maanshan dialect and Mandarin increased significantly from 2000, or 21\%, indicating that the proportion of ethnic minority languages to communicate with people has decreased. This shows that the promotion and penetration rate of Mandarin in China has been greatly improved in the past 10 years, and the promotion and popularization of Mandarin has achieved remarkable results. In daily life, most people have used Mandarin as a daily language, and more and more people speak Mandarin. More people can not only speak Mandarin, but also speak the Maanshan dialect.

\section{The Origin and Development of Maanshan Dialect}

Legend has it that when Chu and Han fought, Xiang Yu, the overlord of Chu, was trapped in Gaixia, defeated and retreated to the bank of the Wujiang River, and entrusted his beloved mount to the fisherman to the other side. He felt that he committed suicide to his father in Jiangdong. WuZhuo horse because of missing the master, rolling self-killing, and the saddle is landed into a mountain, thus, Maanshan name. Maanshan dialect belongs to the Jianghuai mandarin Hongchao. Including now north central Anhui Province: Hefei, Chaohu, Huainan, Bengbu, Chuzhou, Lu'an, etc.; South Anhui: Maanshan, Wuhu, Nanjing, South central Jiangsu, Huaian, Yangzhou, Yancheng, Lianyungang, etc., Nanjing as the representative of Jianghuai mandarin, which is why many people think that Nanjing and Maanshan are very similar, and now Yangzhou as the representative of Jianghuai mandarin.Coupled with its unique geographical location and special cultural history, Maanshan dialect has been formed over time.

Nowadays, after countless years of development, Ma 'Anshan dialect has been very different from other Jianghuai mandarin. Compared with other regional dialects and Mandarin, Maanshan dialect has a phenomenon of nasal separation: en \& eng, in \& ing, $\mathrm{n} \& 1$ and $\mathrm{n} \& \mathrm{l}$, and the words retain rich and obvious characteristics of $\mathrm{Wu}$ dialect.Dialect is the most direct representative of a local cultural characteristics. From some very representative languages below (see the table below), we can see the charm of Maanshan dialect.

\begin{tabular}{ll}
\hline Maanshan dialect & mandarin \\
\hline ge me si & What \\
\hline ge hao qie & How can you eat it? \\
\hline he si ge ren di & To frighten the dead \\
\hline qi hei ma wu di & It's dark outside \\
\hline la qiao & Deliberately ignore, excuse \\
\hline shao & Describe a lot of words to describe a person as conceited \\
\hline da jiang, jie she & Not easy-going, not easy to get along with \\
\hline
\end{tabular}

The people of Maanshan usually add "ah" behind "you (subject)......"

1) For example: Mandarin: Do you (he / she / so) have any money used?

Maanshan dialect: ni a you qian a?

For example: Mandarin: Are you a teacher?

Maanshan dialect: ni a shi lao shi a

2) Maanshan Anshan dialect in the question sentence with "a ....."

The end of the question sentence is "bo".

For example: Mandarin: Is it so?

Maanshan dialect: ge shi zhe yang de bo?

3) Maanshan dialect before and after the nasal sound divided

Like: Mandarin: niu nai. Maanshan dialect: Liu Lai (the third voice)

Mandarin: Can? 
Ma'Shan dialect: Ling bu ling?

"Small away from home boss back, the local sound did not change the temples hair decline", "fellow villagers see fellow villagers, two tears". Indeed, no matter how far away from home, hearing the familiar local sound is the most touching. These dialects may sound rustic, but they really reflect the language characteristics of Ma on Anshan.This special "local sound", through the word of mouth from generation to generation, has become the most prominent hometown characteristic of every Maanshan person, but also embodies the long-term cultural accumulation of the people in their hometown.

\section{Maanshan Dialect Problems in Recent Years}

\subsection{The Influence of Mandarin on the Maanshan Dialect}

The promotion of Mandarin promotes the mutual influence and integration between Mandarin and dialects. With the continuous improvement of the urbanization construction level, the promotion speed of Mandarin is accelerated, and it also has a certain influence on Maanshan dialect.

1). Mandarin, as the common language of China, is all used in TV, movies, newspapers, periodicals, radio and radio stations today, so it is an important carrier of national culture.

2). As the common standard language of the 56 ethnic groups, Mandarin has played an exemplary role in the Maanshan dialect and stipulated the development trend of the dialect.

3). Mandarin has a unified written language, in our daily writing, writing, and even examination have a unified written language, but there is no written language, so in the aspect of written language, the dialect must be affected by Mandarin.

4). China's floating population is large, and people from all regions speak Mandarin when they communicate together, so that it is convenient for their communication and learning. Therefore, the influence of Mandarin will become bigger and bigger, and the population and scope of use are increasing day by day. In this way, the dialect is limited to some extent.

\subsection{The Differences in Some Words Have Become Greater}

Maanshan, as one of the representative core cities of Hefei metropolitan area and Nanjing metropolitan area, is greatly influenced by Mandarin, and within the complex and changeable dialect system. The extension and connotation of many words will change with the development of The Times. The same word in different social periods, its form and function show a high degree of difference. For example: "qie fan" ("eat"), "nai kuai" ("where"), and I often say a rhyme "hun di, huan di, di lan di, le di, bo di, qi he di”" ("red yellow sky blue, green white and black").

\subsection{The Use Frequency Reduces, and the Use Population Is Concentrated in the Old Age}

According to the survey data, only $3.19 \%$ of people currently use dialect completely at work compared to Mandarin, and $47.93 \%$ will choose to use both Mandarin and dialect, and most people still prefer to use Mandarin.In addition, even the indigenous people of Maanshan, many young people rarely use dialect in their daily life.Overall, the population using the dialect was concentrated in the ages born in the 1950s and 1970s.

\section{How to Solve}

\subsection{Protecting and Inheritance of Dialects}

Now in life, the penetration rate of Mandarin has been very high that a small group of young children can not speak their hometown dialect, some children will feel that their hometown dialect is very "local". It is a completely wrong idea. Dialect seems to be used less and less frequently in daily life, and over time, the dialect is eliminated. Therefore, we should actively protect and inherit our dialect.

1. You can carry out local dialect characteristics education to encourage people to popularize Mandarin and do not forget the cultural characteristics of their hometown.

2. Local dialect media can be opened through radio, television and other forms

3. Establish a dialect speech database for each region

\subsection{Build a Harmonious Language Life}

With the development of Chinese society and today's Internet, the cultural and economic exchanges between various regions are also getting closer. People have become more and more frequent contacts, and the circle of friends is expanding rapidly. However, due to its wide region, many ethnic groups, and various dialects, people of ethnic groups in different regions are easy to cause a lot of inconvenience when communicating. Until today, 
China has achieved great results in the promotion and popularization of Mandarin. Mandarin has become a necessary language for people. Even friends thousands of miles apart can communicate in Mandarin. In modern life, the popularity of Mandarin is getting higher and higher, and many parents will set up the awareness of their children speaking Mandarin since childhood. Sign up for small host classes, and the national awareness of Mandarin is increasing day by day. However, as an inseparable part of our daily life, dialect grows with us, contains a unique local culture, and has been integrated into our life. We should combine Mandarin and dialect, and do not forget to inherit our traditional culture while popularizing Mandarin.

\section{Epilogue}

To sum up, we should vigorously promote and popularize Mandarin, but also should not forget our own dialect. No matter how to promote Mandarin in Maanshan, Maanshan is still living in the mouth of people who use the Mandarin. Chatting with family, relatives and friends, go to the vegetable market to buy food, and even some disputes, we always use Mpu. At the same time, other dialects should not die out by the promotion of Mandarin. The popularization of Mandarin has brought convenience to people's lives and made a great contribution to the economic and political development of the country and society. People of 56 nationalities speak the same language, and I think it is a proud thing. While emphasizing the promotion of Mandarin, we should also respect and develop the inheritance of dialect, because it not only represents an accent, but also represents the different historical, cultural and cultural characteristics of each place. We should combine the two perfectly, so that them have become the pride of our nation and even the world.

\section{References}

Chen, B.-Y. (1996). Language Contact and Language Alliance. Beijing: Chinese Press House.

Chen, S.-Z. (1999). Language Variability study. Guangzhou: Guangdong Education Press House.

Chinese Language and Living Status Report Research Group. (2006). The Chinese Language and Living Status Report. Commercial Book Press.

Feng, Z.-W. (1999). Applied linguistics summary. Guangzhou: Guangdong Education Press House.

Han, X.-P. (2000). Chinese Contemporary Reading Theory and Reading Teaching. Chengdu: Sichuan Education Publishing House.

Hou, J.-Y. (2002). An Introduction to the Modern Chinese dialect. Shanghai: Shanghai Education Press House.

National Push General Week Leading Group. (2005). National Publicity Outline of Mandarin Publicity Week (Revised).

Qian, N.-R. (2003). The History of Shanghai Language Development. Shanghai: Shanghai People's Publishing House.

State Council directive on promoting Mandarin. 1956, January 28.

Wang, L.-J., \& Lin, T. (2003). The Voicology Tutorial. Beijing: Peking University Press.

Xu, D.-M., Tao, H.-Y., \& Xie, T.-W. (2004). Contemporary social linguistics. Beijing: China Social Sciences Press.

Yang, B.-J. (2001). Reading Interaction Theory. Education Science, (2).

Yuan, Y. (2001). Language Contact and Language Evolution: Archang Language Case Investigation Study. Beijing: Ethnic Publishing House.

\section{Copyrights}

Copyright for this article is retained by the author(s), with first publication rights granted to the journal.

This is an open-access article distributed under the terms and conditions of the Creative Commons Attribution license (http://creativecommons.org/licenses/by/4.0/). 